psychosis, they have been the focus of little research. Only one theory has been advanced to explain them and this theory has not received consistent empirical support. Recent research has suggested there are two different kinds of delusion of reference: delusions of communication and delusions of observation, and only the latter is associated with hallucinations and persecutory ideation. Delusions of communication may derive from difficulties with self-monitoring, whereby what seems to be communicated concerns the self and originates from the self, although the origin is not recognized but attributed externally.

Methods: This theory was tested with people suffering an acute psychotic episode $(n=63)$ using a visual signal detection task. Participants were required to distinguish between video clips of gestures and nongestures under conditions of uncertainty (visual noise).

Results: The previous finding of two kinds of delusions of reference was replicated. A signal detection analysis showed that people with delusions of communication were no less sensitive to gestures than people with psychosis without these delusions but showed a significantly greater bias toward perceiving them. Whether people had auditory hallucinations or not made no difference to their sensitivity or bias.

Conclusions: It is suggested that bias to perceive gestures occurs because people misattribute selfgenerated events to an external source. However, this bias is not part of a general tendency to externalize one's own thoughts but may be specific to delusions of communication.

\section{Reduced psychoacoustic sensitivity to auditory temporal stimulation in schizophrenia reflects cytoarchitecturally specific changes in auditory cortex}

\section{B Budd}

University of Newcastle, Newcastle, Australia

\begin{abstract}
Aims/Background: The aim of this study was to apply established psychoacoustic and functional magnetic resonance imaging (fMRI) techniques to examine the neuroanatomical basis of auditory temporal processing deficits in schizophrenia. Previously, we showed reduced sensitivity to auditory temporal stimulation using fMRI and psychoacoustic responses to variations in sinusoidally amplitude-modulated noise (SAM). The present study extends these previous findings by examining the extent to which reduced sensitivity to SAM stimulation reflects anatomically specific changes in auditory cortical activity.
\end{abstract}

Methods: Eighteen individuals meeting diagnostic criteria for schizophrenia and 18 controls participated in separate psychoacoustic and fMRI sessions. Regionof-interest analyses were conducted using cytoachitecturally defined anatomical probability maps of primary and secondary auditory cortex. Parametric modulation of auditory BOLD responses was performed using each individual's psychoacoustic SAM detection thresholds for bandpass noise stimuli $(0-6 \mathrm{kHz})$ for $7 \mathrm{SAM}$ rates $(4,8,16,32,64,128$ and $256 \mathrm{~Hz})$.

Results: ROI-based analyses showed that BOLD responses to SAM stimulation were primarily confined to increased activity in transverse temporal gyrus and planum temporal. Analysis of individual sensitivity to SAM rate showed that BOLD responses in anteriorlateral auditory regions showed a significant quadratic function of SAM thresholds. Further, reduced BOLD activity in schizophrenia was evidenced as reduced auditory responses in the same anterior-lateral regions.

Conclusions: The results suggest that reductions in sensitivity to auditory temporal stimulation in schizophrenia may reflect changes in cytoarchitecturally distinct regions of primary auditory cortex (te1.2). These findings are discussed in terms of the possible neural mechanisms underlying auditory temporal processing deficits in schizophrenia.

\section{Mental health problems within couples and marital disruption}

\section{P Butterworth}

Centre for Mental Health Research, The Australian National University, Canberra, Australia

Background: There is growing attention to the social consequences of psychiatric disorders, including the extent to which psychiatric disorders are associated with subsequent divorce and relationship dissolution. The previous research has largely studied individuals and given only limited consideration to the broader context of marriage and the potential interplay between spouses' mental health. The current paper studies couples and examines the association between both spouses' mental health problems and subsequent marital dissolution.

Methods: Prospective analysis of secondary data from a longitudinal national household survey. About 3230 couples were followed over 36 months, with logistic and multilevel regression models used to determine whether mental health problems of spouses at wave 1 (assessed by the mental health scale of SF-36) predicted subsequent marital dissolution.

Results: Couples in which either men or women reported mental health problems had higher rates of marital disruption than couples in which neither spouse 